\title{
Accelerating innovation in medicine: a wake-up call amidst the COVID-19 pandemic
}

\author{
Janny Xue Chen Ke, MD, FRCPC (1) - Alexander Waslen, BE (Mech) • Chance Park, BSc • \\ Orlando Hung, MD, FRCPC
}

Received: 18 July 2021 / Revised: 2 August 2021 / Accepted: 2 August 2021 / Published online: 17 August 2021

(C) Canadian Anesthesiologists' Society 2021

Keywords innovation - innovators - medical inventions . medical innovation $\cdot$ innovation regulation

Foreseeing a potential shortage of ventilators as the COVID-19 pandemic spread to Canada, in March 2020, Dr. Christopher Nguan, a urologist at the University of British Columbia (UBC), brought together a team to design a low-cost, open-source ventilator. This interdisciplinary group of volunteers formed the non-profit Collective Open Source Medical Innovations for COVID-19 (COSMIC), ${ }^{1}$ and won a national challenge award of CAD 100,000 from Roche Canada for further development of the ventilator. COSMIC has since grown to pursue additional low-cost innovations. A Bubble Helmet interface to deliver comfortable positive pressure ventilation has been approved by Health Canada for clinical trials and is in the process of scaling manufacturing for distribution. Similarly, Canadian anesthesiologists have been

\section{J. X. C. Ke, MD, FRCPC ( ()}

Department of Anesthesia, St. Paul's Hospital, Providence

Health Care, Vancouver, BC, Canada

e-mail: jannyke@interchange.ubc.ca

Department of Anesthesiology, Pain Management, and Perioperative Medicine, Dalhousie University, Halifax, NS, Canada

A. Waslen, BE (Mech)

Department of Mechanical Engineering, University of British

Columbia, Vancouver, BC, Canada

C. Park, BSc

Department of Medicine, University of British Columbia,

Vancouver, BC, Canada

O. Hung, MD, FRCPC

Department of Anesthesiology, Pain Management, and Perioperative Medicine, Dalhousie University, Halifax, NS, Canada investigating devices to protect frontline healthcare workers, such as a face tent for aerosol evacuation ${ }^{2}$ and a negative pressure barrier for extubation. ${ }^{3}$

During the COVID-19 pandemic, medical innovations have been advancing at an accelerated pace. This has been in part driven by dire necessity and supported by an unprecedented level of collaboration across institutions, governments, industries, and regulatory agencies. Examples include rapid vaccine development and deployment, ${ }^{4}$ digital health technology adoption, and completion of international multicentre research trials. Thus, the COVID-19 crisis has given us an opportunity to re-examine many processes, including the optimal idea-toimplementation pathways for innovations. If research and regulation can be accelerated without compromising effectiveness and safety during a pandemic, could this be the post-pandemic model that leads us into the future?

\section{Barriers and opportunities for innovation}

Physicians are a vital source of medical innovation because they have a deep understanding of issues and solutions in the clinical setting. Anesthesiologists, in particular, encounter diverse aspects of surgery and medicine, and bring unique perspectives at both patient and systems levels. Nevertheless, the path to implementing innovation is plagued with hurdles. Overcoming these old hurdles requires new strategies.

Streamlined pathway for innovation

As a culture, medicine is resistant to change, and deviations from the status quo are often discouraged. This is in part a necessity due to the crucial pillars of patient safety, quality of care, medical-legal regulations, and ethical obligations. Without proper evaluation and risk mitigation, innovations can bring unintended consequences. For example, upon 
further testing, some barrier enclosures designed to reduce exposure to aerosolized virus particles during intubation (an aerosol-generating medical procedure) were found to make airway management more challenging with no evidence of decreased risk of transmission for the healthcare provider. $^{5}$ Thus, all medical innovations must undergo rigorous clinical testing and regulatory evaluation to ensure safety and effectiveness. Well-designed, blinded multicentre randomized controlled trials should be the norm, in contrast to the current reliance on small observational studies for medical equipment and devices.

Nevertheless, to bring creative ideas to fruition in a timely fashion, there are many opportunities to reform the existing labyrinthine process. ${ }^{6}$ The completion of multiple large multicentre randomized trials related to COVID-19 showed us that it is logistically possible to complete these studies in time scales much shorter than the pre-pandemic norm. In addition, along with many other jurisdictions, Health Canada created special pathways during the pandemic to safely expedite the regulatory process. ${ }^{7} \mathrm{We}$ should learn from these experiences to develop best practices, and address the inefficiencies and bottlenecks in the research and regulatory pipeline. Coordinated efforts among governments, research institutions, and industries are required to implement streamlined pathways and collaborative networks for rigorous, efficient evaluation of medical innovations. ${ }^{6}$

\section{An ecosystem for innovation}

Having an idea to address a problem is only the first step. From engineering, product design, protection of intellectual property, clinical testing, and regulatory evaluation to commercialization, there are many critical aspects of bringing a product to patients that are beyond physicians' expertise. Many physician innovators work in silos and have limited organizational support to succeed, including funding, protected time for research, and mentorship.

An interdisciplinary team is needed to push innovations through to implementation. The success of COSMIC can be partly attributed to the diverse team, which included healthcare professionals, designers, engineers, and lawyers. Other support from the Canadian medical community included training and grants from Joule at the Canadian Medical Association and mentorship and network from Physician Innovator (physicianinnovator.com). Moving forward, we need a more sustainable infrastructure to guide, mentor, and support physician innovators, while addressing barriers in diversity, equity, and inclusion. We must learn from institutions with best practices, expand interdisciplinary collaborations, and increase the accessibility of resources. Importantly, we need to engage patients in co-creation of patient-centred innovations. Integration of funding, mentored incubators, and regional and national networks of manufacturing and distribution will be crucial in supporting physician innovators from inception through to commercialization. ${ }^{8}$

\section{Funding}

In contrast to the rest of the $\mathrm{G} 7$ countries, Canada's gross domestic spending on research and development has been dwindling since 2001. ${ }^{9}$ In 2016-2017, the American National Institutes of Health budget for medical research was USD 30.6 billion, compared with the Canadian Institutes of Health Research budget of only CAD 1.03 billion. ${ }^{9}$ Following years of underinvestment in research and development, we should not be surprised to witness that Canada lagged behind all G7 countries in development of a COVID-19 vaccine. Learning from this, government, academia, and industry need to collaborate more to invest in a sustainable research and development infrastructure that will be resilient to future threats. Existing funding models include the British Columbia Digital Supercluster, the Nova Scotia Early Stage Commercialization Fund, TEC Health Accelerator, and the Angel Investor Tax Credit. ${ }^{8}$ In addition to supporting existing programs, seed funding is needed to encourage proof-of-concept exploration and experimentation, such as the Innovative Solutions Canada. Another source is the non-profit sector, such as the Bill and Melinda Gates Foundation. To make funding more efficient, we need to better coordinate research priorities $^{10}$ on a national policy level and integrate research and clinical care. ${ }^{11}$

\section{Training and education}

As shown in the pandemic, a physician's key skill set involves thinking critically and creatively when facing challenges, while rapidly adapting to new changes. Building a future physician workforce with this ability starts with the selection of students who have an interest in creative thinking and innovation, and teaching the innovation process throughout medical training and continuing medical education. The winning entry of the 2016 American Medical Association Medical Education Innovation Challenge proposed incorporation of new skills such as computer science and small electronics in the preclinical curriculum. ${ }^{12}$ At a grassroots level, initiatives to increase opportunities for innovation include the UBC "Hatching Health," where students from medicine, allied health students, engineering, design, and computer science streams work together to create an innovation. While similar initiatives exist in medical schools, innovation and entrepreneurship education for the medical community 
need to be more widely integrated. Ideas include a formalized curriculum, as well as innovation tracks during medical school, residency, and fellowship. Planning for the future, we need to emphasize through policy that innovation is a complex process that requires continued mentorship, resources, and training, and needs to be systematically nurtured and incentivized throughout a physician's career.

\section{Conclusion}

During the COVID-19 pandemic, in addition to dedicated clinical service, many physicians have been developing and evaluating innovations to help patients. While the pandemic has exposed our weaknesses in medical innovation, such as limited infrastructure and funding, it has highlighted our strengths including creativity and collaborative networks. To foster future physician innovation, more streamlined pathways, organizational support, funding, and formalized training are needed. The medical community should take advantage of the current momentum and accelerated pace of innovations, and advocate for the necessary policy changes to help us meet future challenges within our new paradigm.

Acknowledgements We would like to thank Dr. Christopher Nguan for his feedback on the manuscript.

Disclosures Alexander Waslen is a Board Member of COSMIC Medical Non-profit. Chance Park is the Founding President of Physician Innovators. Orlando Hung holds patents on FIVA ${ }^{\mathrm{TM}}$, a light-guided intubation device, and pulmonary delivery of liposomal encapsulated opioids and cannabinoids.

Funding statement None.

Editorial responsibility This submission was handled by Dr. Philip M. Jones, Deputy Editor-in-Chief, Canadian Journal of Anesthesial Journal canadien d'anesthésie.

\section{References}

1. COSMIC Medical. Collective Open Source Medical Innovations of COVID-19; 2020. Available from URL: https:// cosmicmedical.ca/ (accessed August 2021).

2. Tsui BC. Re-purposing a face tent as a disposable aerosol evacuation system to reduce contamination in COVID-19 patients: a simulated demonstration. Can J Anesth 2020; 67: 1451-3.

3. Hung $O$, Hung $D$, Hung $C$, Stewart R. A simple negative-pressure protective barrier for extubation of COVID-19 patients. Can J Anesth 2020; 21: 1-3.

4. Ball $P$. The lightning-fast quest for COVID vaccines - and what it means for other diseases. Nature 2020; 589: 16-8.

5. Sorbello $M$, Rosenblatt $W$, Hofmeyr $R$, Greif $R$, Urdaneta $F$. Aerosol boxes and barrier enclosures for airway management in COVID-19 patients: a scoping review and narrative synthesis. $\mathrm{Br}$ J Anaesth 2020; 125: 880-94.

6. Stewart J, Honig P, AlJuburi L, et al. COVID-19: A catalyst to accelerate global regulatory transformation. Clin Pharmacol Ther 2020; DOI: https://doi.org/10.1002/cpt.2046

7. Government of Canada. Health Canada's regulatory response to COVID-19: Access to health products. Available from URL: https://www.canada.ca/en/health-canada/services/drugs-health-products/ covid19-industry/regulatory-response-health-product-access.html (accessed August 2021).

8. MacNeil M, Koch M, Kuspinar A, Juzwishin D, Lehoux P, Stolee $P$. Enabling health technology innovation in Canada: barriers and facilitators in policy and regulatory processes. Health Policy 2019; 123: 203-14.

9. Canadian Association for Neuroscience. Science funding in Canada - Statistics. Available from URL: https://can-acn.org/ science-funding-in-canada-statistics/ (accessed August 2021).

10. McKeen DM, Banfield JC, McIsaac DI, et al. Top ten priorities for anesthesia and perioperative research: a report from the Canadian Anesthesia Research Priority Setting Partnership. Can J Anesth 2020; 67: 641-54.

11. Angus DC, Gordon AC, Bauchner H. Emerging lessons from COVID-19 for the US clinical research enterprise. JAMA 2021; DOI: https://doi.org/10.1001/jama.2021.3284

12. American Medical Association. AMA advances initiative to create the medical school of the future; 2016. Available from URL: https://www.ama-assn.org/press-center/press-releases/amaadvances-initiative-create-medical-school-future (accessed August 2021).

Publisher's Note Springer Nature remains neutral with regard to jurisdictional claims in published maps and institutional affiliations. 